(C) 1982. The Genetical Society of Great Britain

\title{
THE CHROMOSOMAL DISTRIBUTION OF CLONED HIGHLY REPETITIVE SEQUENCES FROM HEXAPLOID WHEAT
}

\author{
J. HUTCHINSON and D. M. LONSDALE \\ Plant Breeding Institute, Cambridge, U.K.
}

Received 19.xii.81

\begin{abstract}
SUMMARY
Two highly repetitive DNA sequences from Triticum aestivum were isolated and purified by molecular cloning. In situ hybridisation of these sequences to wheat chromosomes showed the sequence to be located predominantly at specific sites in the $B$ genome and also chromosome $4 A$. Individual chromosomes could be identified by their patterns of labelling. Although one sequence was found to be mostly located close to centromeres and interstitially, and the other sequence mostly at telomeres, there was a good correlation between the distribution of these sequences and the distribution of heterochromatin as revealed by C-banding.

In situ hybridisation of the two sequences to chromosomes of the related species, Secale cereale and Hordeum vulgare showed a similar correlation between the location of highly repetitive sequences and heterochromatin.
\end{abstract}

\section{INTRODUCTION}

IN common with many other plants, a large proportion of the genome of hexaploid wheat (Triticum aestivum) consists of repetitive sequence DNA (Smith and Flavell, 1974; 1975). Flavell et al. (1979) isolated a fraction from the wheat genome enriched for the most highly repeated sequences and from a series of filter hybridisations to DNAs from different diploid and tetraploid species related to hexaploid wheat, concluded that the most highly repetitive DNA of wheat was localised in the $B$ genome rather than in the $A$ or $D$ genomes. This was confirmed by finding that the highly repetitive fraction isolated from a species closely related to the $B$ genome (Aegilops speltoides) showed only weak hybridisation to various $\boldsymbol{A}$ genome diploid species, and a substantially reduced level of hybridisation to $A e$. squarrosa (Genome $D D$ ).

The highly repetitive fractions contained many different families of repetitive sequences. To analyse the fraction in more detail, and in particular the chromosomal distribution of individual families in the wheat genome, members of the predominant families were purified by molecular cloning. The cloned sequences were selected from a small bank of clones of wheat DNA restricted with HaeIII, by their strong hybridisation to the highly repetitive fraction from both Triticum aestivum and Aegilops speltoides. Most of the cloned sequences selected belonged to two families of repeated sequences. The distribution of these highly repeated sequences in both wheat and in rye (Secale cereale) and barley (Hordeum vulgare) and the relationship with heterochromatin is the subject of this paper. 


\section{MATERIALS AND METHODS}

(i) Plant stocks

The following plant stocks were used: Triticum aestivum cv. Chinese Spring $(2 n=6 x=42)$ and its appropriate telocentric and double ditelocentric lines; T. monococcum acc. A $(2 n=2 x=14)$; Aegilops sharonensis acc. A $(2 n=2 x=14)$; Ae. squarrosa acc. A $(2 n=2 x=14)$; Secale cereale cv. Petkus Spring and cv. UC90 $(2 n=2 x=14)$ and Hordeum vulgare cv. Sultan and $c v$. Sundance $(2 n=2 x=14)$.

\section{(ii) In situ hybridisation}

Root-tips were prepared and the in situ hybridisation carried out as described (Hutchinson et al., 1980). After developing the autoradiographs, the slides were stained either in Carbol Fuchsin stock stain (Darlington and La Cour, 1969) or in 6 per cent aqueous Giemsa stain, before being mounted in immersion oil.

\section{(iii) Radioactive $R N A$ probes}

The probes used were tritium labelled cRNA's transcribed by $E$. coli RNA polymerase (Gall and Pardue, 1971) from chimaeric plasmids. The source of the DNA used to produce the clones was T. aestivum cv. Chinese Spring and the construction and organisation of the clones is described in detail by Lonsdale and Hutchinson (in preparation). Brief details of the probes used in the present investigations are tabulated below:

\begin{tabular}{lcc}
\multicolumn{1}{c}{ Probe } & Plasmid vector & $\begin{array}{c}\text { Wheat DNA } \\
\text { sequence length }\end{array}$ \\
pCS(1)TC22b & M13 & $0.25 \mathrm{~Kb}$ \\
pCS(1)C40 & pACY184 & $1.70 \mathrm{~Kb}$
\end{tabular}

\section{Results}

(i) Chromosomal locations of highly repetitive sequence probes in wheat

Two clones were derived from a genome clone bank of Triticum aestivum cv. Chinese Spring. These clones, pCS(1)TC22b and pCS(1)C40, (abbreviated in the text to TC22b and $\mathrm{C} 40$ respectively) were selected as containing highly repetitive sequences, probably from the $B$ genome of wheat.

\section{(a) Chromosomal location of TC22b}

In situ hybridisation of probe $\mathrm{TC} 22 \mathrm{~b}$ to the mitotic chromosomes of Chinese Spring wheat showed the sequence to be located predominantly in blocks on eight pairs of chromosomes (fig. 1). The identity of the labelled chromosomes was determined firstly by looking at the hybridisation of the probes to the diploid species thought to be the progenitors of hexaploid wheat. It is generally accepted that hexaploid wheat (Genome $A A B B D D$ ) originated as a hybrid between a tetraploid wheat (Genome $A A B B$ ) and Aegilops squarrosa (Genome $D D$ ) (McFadden and Sears, 1946; Riley and Chapman, 1960). As shown in fig. 2(a), Ae. squarrosa shows little hybridisa- 


\section{Plate I}

A

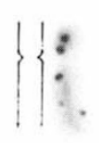

2

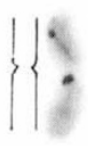

3

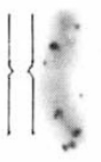

4
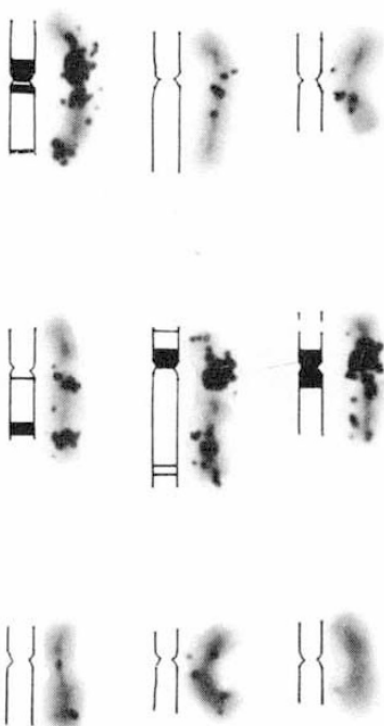

6

7

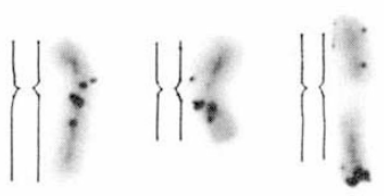

B
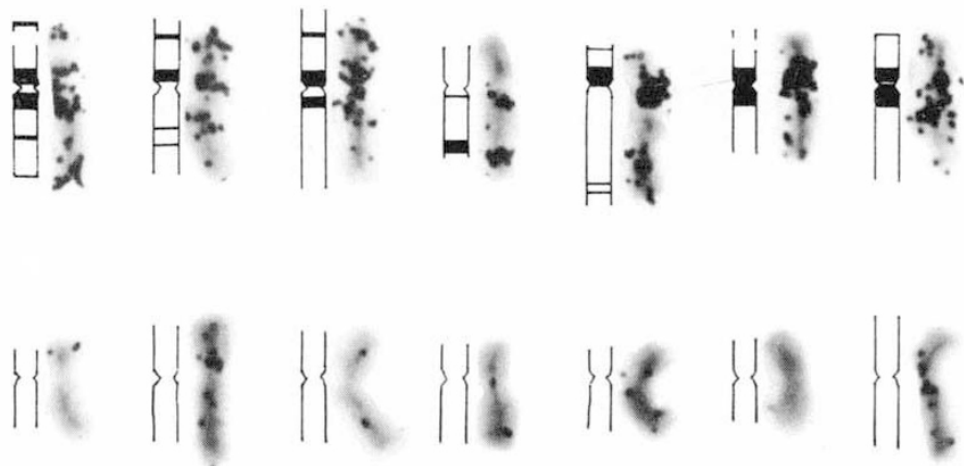

FIG. 1.-Idiogram of the pattern of labelling of hexaploid wheat, cv. Chinese Spring, following in situ hybridisation of the TC22b probe. The heavily labelled chromosomes were identified by examining the labelling patterns in many cells of replicated in situ hybridisations to mitotic chromosome preparations of telocentric stocks of Chinese Spring. The labelling of the other chromosomes was slight, and so chromosomes were identified only by their size and arm ratios, and not by examining telocentric stocks. Since this is not a precise method of identifying chromosomes, lines were not drawn on the idiograms of these chromosomes. The karyotype is based on measurements made of chromosome lengths and arm ratios by Sears (1954). Also shown in the figure are photographs taken from one cell, of the typical labelling patterns obtained after hybridisation with the TC22b probe. 750,000 c.p.m. of probe were applied per slide and the autoradiographs exposed for 1 month. 
Plate II

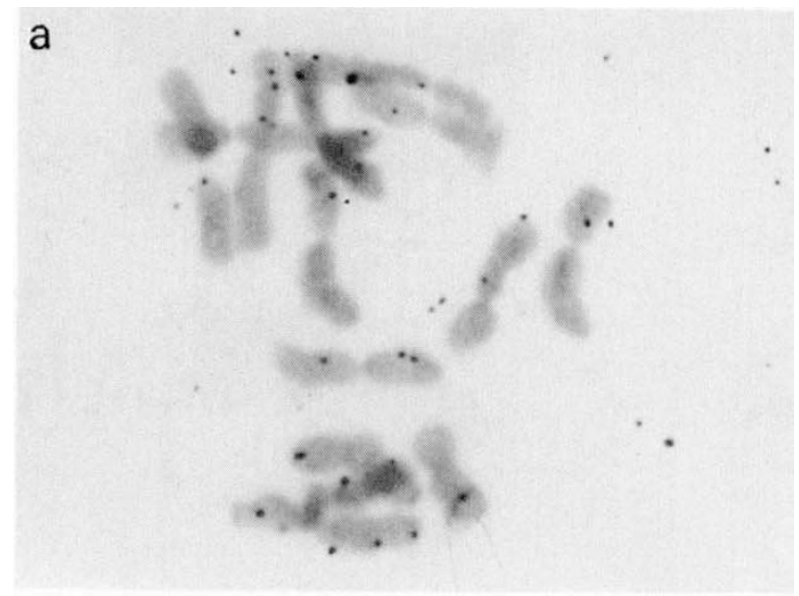

b
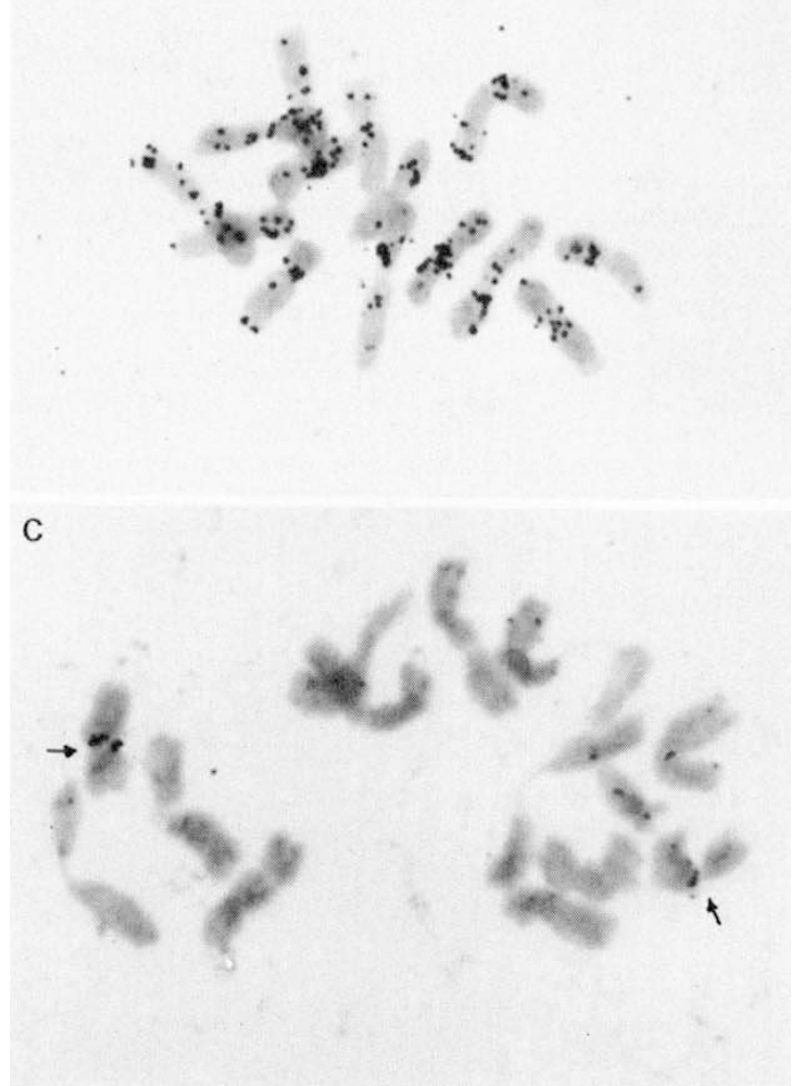

FIG. 2.-In situ hybridisation of the TC22b probe to mitotic chromosomes of: (a) Aegilops squarrosa (Genome $D D$ ), (b) Aegilops sharonensis (Genome $S S$, related to the $B$ genome), (c) Triticum monococcum (Genome $A A$ ). 
tion with TC22b. Similarly, $T$. monococcum, which has the $A A$ genome, shows only a low level of hybridisation, although with longer exposure times, it is possible to show that the probe does hybridise to the centromeric regions of one pair of chromosomes (fig. 2(c)). Similarly, only low levels of hybridisation have been observed in other $A$ genome diploid wheats. Although the donor of the $B$ genome of wheat is a matter of some debate, it is thought that members of the Sitopsis section of the genus Aegilops are closely related to the $B$ genome of tetraploid and hexaploid wheats (see Feldman, 1978). Ae. sharonensis, which is a member of this Sitopsis group, shows very heavy labelling with the probe TC22b (fig. 2(b)). This therefore implies that, as expected, most of the labelled chromosomes belong to the $B$ genome of hexaploid wheat.

To confirm this, and also to establish the identity of the eighth pair of labelled chromosomes, telocentric stocks of Chinese Spring were probed with TC22b. The results showed that major sites of the sequence occurred in all the $B$ genome chromosomes, and also on chromosome $4 A$.

As shown in fig. 1, TC22b is found predominantly either near the centromeres or at interstitial sites with very little telomeric localisation, and it is possible to identify individual chromosomes by their patterns of labelling. These patterns, with one slight exception described later, paralleled very closely the distribution of a satellite DNA isolated from wheat and barley using $\mathrm{Ag}^{+} / \mathrm{Cs}_{2} \mathrm{SO}_{4}$ buoyant density gradients (Density = $1 \cdot 705 \mathrm{~g} \cdot \mathrm{ml}^{-1}$ ) (Gerlach et al., 1978; Dennis et al., 1980), and also a satellite sequence from Drosophila melanogaster (Density in $\mathrm{CsCl}$ $1 \cdot 705 \mathrm{~g} \mathrm{.} \mathrm{ml}^{-1}$ ) (Appels and Peacock, 1978; May and Appels, 1980). TC22b does not cross hybridise under stringent conditions with the clones of the silver satellite sequences which were developed by Gerlach et al. (1981). This therefore implies that two different highly repetitive DNA sequences are located close to each other in wheat chromosomes. This was predicted by Gerlach and Peacock (1980) who found a similar pattern of distribution when they probed wheat chromosomes with $C_{0} t 10^{-2}$ DNA, which, of course, comprises many different repeating sequences. In addition, they found that this labelling by the $C_{0} t 10^{-2}$ fraction was not diminished by the presence of unlabelled silver satellite competitor DNA, indicating that other sequences must have contributed to the overall labelling pattern.

One slight difference in the pattern of labelling observed when probing with TC22b versus the sitver satellite sequence, involved chromosome $7 A$. Using the silver satellite sequence probe, chromosome $7 A$ is labelled at both telomeres (Gerlach et al., loc. cit.), whereas the chromosome is only weakly labelled at one telomere by the TC22b probe. This result can be explained in two ways. One possibility is that the different sequences are not invariably associated together. The other possibility is that there is variation between the different stocks of Chinese Spring wheat used for the in situ hybridisations in Australia and England. Indeed this explanation is likely because different Chinese Spring stocks show variation in telomeric heterochromatin (Seal, personal communication).

\section{(b) Chromosomal location of probe $\mathrm{C4O}$}

In situ hybridisation of $\mathrm{C} 40$ to mitotic chromosomes of Chinese Spring again showed eight pairs of labelled chromosomes (fig. 3). By probing firstly 
the diploid progenitor species (fig. 4) and secondly telocentric lines, it was again possible to establish that most of the labelling was confined to the $B$ genome and $4 A$ chromosomes. The label was largely limited to telomeric and occasional interstitial sites however, in contrast to the pattern of labelling shown by TC22b (fig. 1). Similar telomeric labelling of wheat chromosomes has previously been reported by Bedbrook et al. (1980) for a highly repetitive $120 \mathrm{bp}$ sequence from rye purified by molecular cloning. Filter hybridisations have shown some degree of cross hybridisation between this rye 120 bp repeat and C40, indicating that they must share a common sequence, and so that similar patterns of chromosome labelling may have been due to hybridisation with this common sequence.

\section{(ii) Comparison of the distribution of repetitive sequence DNA with heterochromatic bands}

Examination of wheat chromosomes by means of $\mathrm{C}$-banding and $\mathrm{N}$ banding techniques have shown the $B$ genome and $4 A$ chromosomes to be the most heterochromatic (Gill and Kimber, 1974; Gerlach, 1977). $\mathrm{N}$-banding is an alternative technique for revealing heterochromatin following a hot acid treatment, compared with the Barium-hydroxide and SSC treatment of the C-banding technique.

The banding patterns of individual chromosomes have been well characterised, and can be used for chromosome identification. As illustrated in fig. 5, most of the major heterochromatic bands are located centromerically and interstitially, and there is a very close resemblance between the patterns of labelling shown by probe TC22b and the heterochromatic bands. At first sight it appears that the distribution of the $\mathrm{C} 40$ probe, which is largely telomeric or interstitial, does not correspond with the heterochromatin. However, from the published karyotypes it may be seen that while the major heterochromatic bands revealed by $\mathrm{C}$ - or $\mathrm{N}$-banding are essentially similar, a few more minor bands are obtained with the $\mathrm{C}$-banding technique (fig. 5). The distribution of the highly repetitive $\mathrm{C} 40$ probe corresponds with the distribution of some of these minor bands. This finding is not unexpected, since various studies have demonstrated the association between tandemly arranged, highly repetitive DNA sequences and constitutive heterochromatin (Appels et al., 1978; see also review by Appels and Peacock, 1978).

(iii) Localisation of wheat highly repetitive sequences in closely related species

(a) Rye

In situ hybridisation of the $\mathrm{TC} 22 \mathrm{~b}$ probe to mitotic rye chromosomes showed a slight scatter of silver grains on several chromosomes, together with a localised site near the centromeres on one pair of chromosomes (fig. 6(a)). This result contrasts with the data published for the silver satellite sequence, which was not found to hybridise to rye chromosomes (May and Appels, loc. cit.). However, both the scattered and localised sites of TC22b were only revealed when fairly high c.p.m. of probe were applied to the slides, and when the autoradiographs were exposed for long periods. 


\section{Plate III}

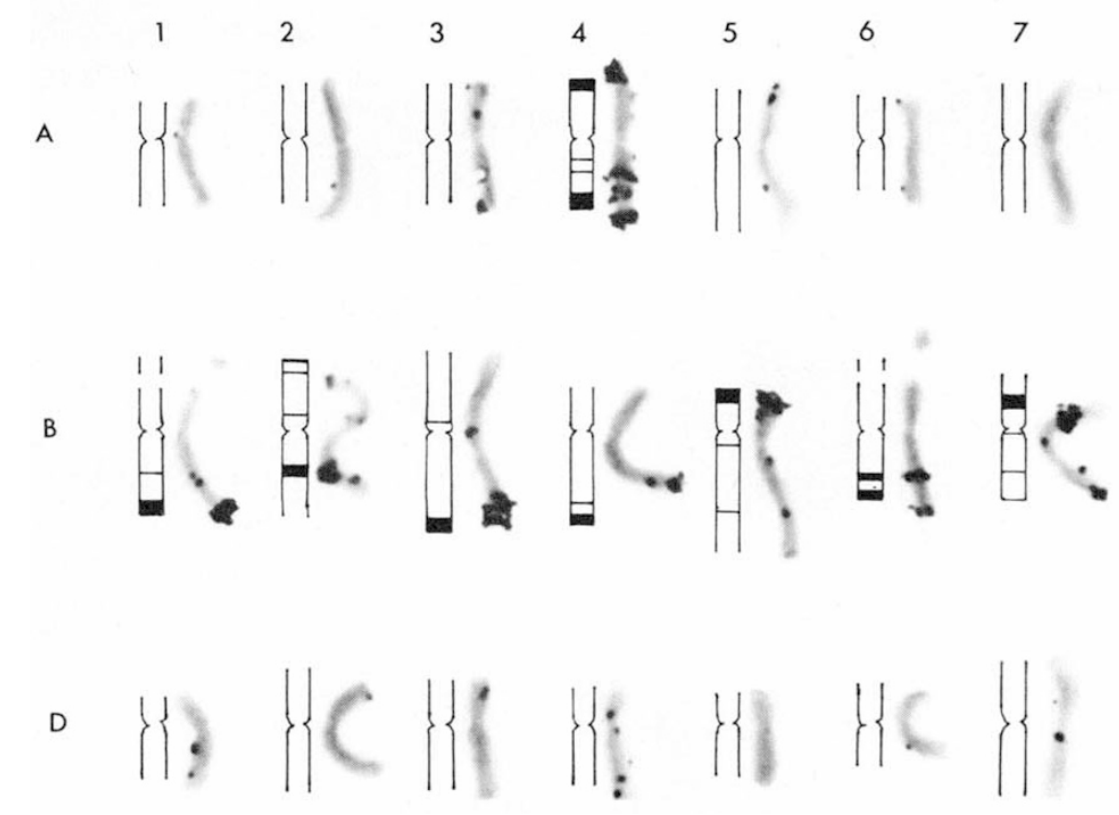

FIG. 3.-Idiogram of the labelling patterns of hexaploid wheat, cv. Chinese Spring, following in situ hybridisation with the $\mathrm{C} 40$ probe. Also shown are photographs taken from one cell, showing the typical labelling patterns observed with this C40 probe. The chromosomes were identified, and the idiograms prepared as described in the legend to fig. 1. 380,000 c.p.m. were applied per slide, and the autoradiographs exposed for 4 months. 


\section{Plate IV}

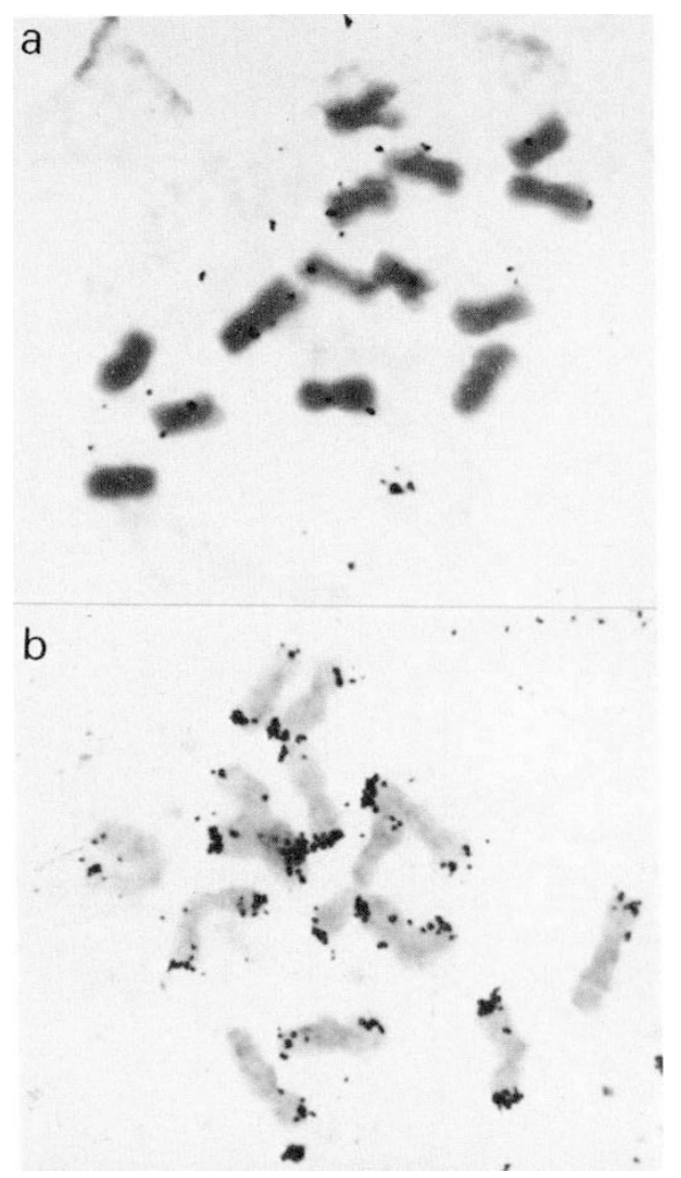

FIG. 4.-In situ hybridisation of the C40 probe to mitotic chromosomes of: (a) Triticum monococcum (Genome AA), (b) Aegilops sharonensis (Genome SS). 
Preliminary Southern Blot data also suggest that the silver satellite sequence may be present in rye in a low copy number (Jones, 1980). In contrast to these lower copy numbers of both the TC22b and silver satellite sequence probes, the $\mathrm{C} 40$ sequence is present in quite high copy numbers in the rye genome. In situ hybridisations show the sequence is located at both telomeric and interstitial sites (fig. 6(b)). These sites probably correspond with the sites of hybridisation shown by the 120 bp repeat of rye (Bedbrook et al., loc. cit.; Jones and Flavell, loc. cit.) since as mentioned earlier, C40 has been shown to cross hybridise with this $120 \mathrm{bp}$ repetitive DNA sequence from rye.

\section{(b) Barley}

In situ hybridisation of the $\mathrm{C} 40$ probe to mitotic chromosomes of barley showed very little hybridisation (fig. 6(c)). In contrast, the TC22b probe showed strong hybridisation to the regions near the centromeres (fig. 6(d)). In barley it has been shown that most of the heterochromatin is located near the centromeres of the chromosomes (Linde-Laursen, 1975; Vosa, 1976). This therefore again demonstrates the correlation between the distribution of highly repetitive sequences and constitutive heterochromatin.

\section{Discussion}

In situ hybridisation of the highly repetitive sequence probes TC22b and $\mathrm{C} 40$ to the chromosomes of wheat has shown the sequences to be located predominantly at particular sites in the $B$ genome and $4 A$ chromosomes. The TC22b sequence is located mainly centromerically and interstitially, while in contrast the $\mathrm{C} 40$ sequence is located mainly at the telomeres. The distribution of both sequences is however closely correlated with the distribution of heterochromatin as revealed by $\mathrm{C}$-banding.

In addition, the in situ hybridisations of the wheat highly repetitive sequences to related species also shows that certain sequences tend to be limited to particular regions of the chromosome. In other words, sequences which are found at the telomeres of one species, if present at all, tend to be located also at the telomeres in other species. The same is true for the localisation of the interstitial or centromeric sequences. The simplest explanation for this finding is that this is a residual effect resulting from the common ancestry of wheat, rye and barley. However, it is also possible that there are also certain chromosomal domains which are limited to particular highly repetitive sequences, either because amplification of only certain sequences is possible, or because deletion of other sequences occurs. If this is a common phenomenon it could well throw some light on the role of tandemly arranged highly repetitive sequences. It will, however, be necessary to look at the distribution of many more such sequences in more species before firm conclusions can be made. 


\section{REFERENCES}

APPELS, R., DRISCOLL, C. J., AND PEACOCK, W. J. 1978. Heterochromatin and highly repeated DNA sequences in rye (Secale cereale). Chromosoma (Berl.), 70, 67-90.

APPELS, R., AND PEACOCK, W. J. 1978. The arrangement and evolution of highly repeated (satellite) DNA sequences with special reference to Drosophila. Int. Rev. Cytol. Suppl., 8, 69-126.

BEDBROOK, J. R., JONES, J., O'DELL, M., THOMPSON, R. D., AND FLAVELL, R. B. 1980. A molecular description of telomeric heterochromatin in Secale species. Cell, 19, 545-560.

DARLINGTON, C. D., AND LaCOUR, L. F. 1969. The Handling of Chromosomes. George Allen and Unwin, Ltd.

DENNIS, E. S., GERLACH, W. L., AND PEACOCK, W. J. 1980. Identical polypyrimidine polypurine satellite DNA in wheat and barley. Heredity, 44, 345-366.

FEldman, M. 1978. Origin of the $B$ genome. In Ramanujam, S. (ed.) Proc. 5th Int. Wheat Genet. Symp., vol. 1, pp. 120-132.

FLAVELL, R. B., O'DELL, M., AND SMITH, D. B. 1979. Repeated sequence DNA comparisons between Triticum and Aegilops species. Heredity, 42, 309-322.

GALL, J. G., AND PARDUE, M. L. 1971. Nucleic acid hybridization in cytological preparations. In Grossman and Moldave (eds.) Methods in Enzymology, Academic Press, New York.

GERLACH, W. L. 1977. N-banded karyotypes of wheat species. Chromosoma (Berl.), 62, 49-56.

GERLACH, W. L., APPELS, R., DENNIS, E. S., AND PEACOCK, w. J. 1978. Evolution and analysis of wheat genomes using highly repeated DNA sequences. In Ramanujam, S. (ed.) Proc. 5th Int. Wheat Genet. Symp., vol. 1, pp. 81-91.

GERLACH, W. L., AND PEACOCK, W. J. 1980. Chromosomal locations of highly repeated DNA sequences in wheat. Heredity, 44, 269-276.

GERLACH, W. L., DENNIS, E. S., AND PEACOCK, W. J. 1981. Molecular cytogenetics of wheat. In Swaminathan, M. S., Gupta, P. K. and Sinha, H., (eds) Cytogenetics of Crop Plants, in press.

GILL, B. S., AND KIMBER, G. 1974. Giemsa C-banding and the evolution of wheat. Proc. nat. Acad. Sci. (Wash.), 71, 4086-4090.

HUTCHINSON, J., CHAPMAN, V., AND MILlER, T. E. 1980. Chromosome pairing at meiosis in hybrids between Aegilops and Secale species: a study by in situ hybridisation using cloned DNA. Heredity, 45, 245-254.

JONES, J. 1980. Repeated DNA sequences in rye (Secale cereale), wheat (Triticum aestivum) and their relatives. Ph.D. Thesis, University of Cambridge.

LINDE-LAURSEN, I. 1975. Giemsa C-banding of the chromosomes of "Emir" barley. Hereditas, 81, 285-289.

MAY, C. E., AND APPELS, R. 1980. Rye chromosome translocations in hexaploid wheat: a re-evaluation of the loss of heterochromatin from rye chromosomes. Theor. Appl. Genet., $56,17-23$.

MCFADDEN, E. S., AND SEARS, E. R. 1946. The origin of Triticum spelta and its free threshing relatives. $J$. Hered., 37, 81-116.

RILEY, R., AND CHAPMAN, v. 1960. The $D$ genome of hexaploid wheat. Wheat Inf. Service, $11,18-19$.

SEARS, E. R. 1954. The aneuploids of common wheat. Missouri Agr. Exp. Sta. Res. Bull., 572, 3-59.

SMITH, D. B., AND FLA VELL, R. B. 1974. The relatedness and evolution of repeated nucleotide sequences in the genomes of some Gramineae species. Biochem. Genet., 12, 243-256.

SMITH, D. B., AND FLAVELL, R. B. 1975. Characterisation of the wheat genome by renaturation kinetics. Chromosoma (Berl.), 50, 223-242.

vosA, C. G. 1976. Chromosome banding patterns in cultivated and wild barleys (Hordeum spp.). Heredity, 37, 395-403. 